\title{
Enabling Technologies for High Performance Millimetre and Sub-millimetre Wave Radar
}

\author{
Duncan A. Robertson* \\ *University of St Andrews, School of Physics \& Astronomy \\ St Andrews, SCOTLAND \\ email: dar@st-and.ac.uk
}

\begin{abstract}
The successful realisation of high performance millimetre and sub-millimetre wave radars requires key enabling technologies, many of which are not yet commercially available. This paper illustrates some of the key enabling technologies developed to address radar system requirements including chirp generation, feedhorns, duplexing and non-mechanical beam steering. The type of high performance radar system which can be achieved using these technologies is illustrated with the examples of the 'T-220' $94 \mathrm{GHz}$ FMCW Doppler radar used for high sensitivity target and clutter phenomenology studies and the 'CONSORTIS' $340 \mathrm{GHz} 3 \mathrm{D}$ imaging radar developed for concealed object detection as required for next generation aviation security screening.
\end{abstract}

\section{Introduction}

Radars which operate in the millimetre and sub-millimetre wave spectrum offer particular advantages arising from the trade-offs between achievable spatial and range resolutions, Doppler sensitivity, penetration through obscurants and system size. Applications for radars at these frequencies are very diverse and include security screening [1], material inspection [2], helicopter powerline detection and brownout collision avoidance [3], [4], and atmospheric sensing [5], [6]. The successful realisation of sub/millimetre wave radars depends on the availability of enabling technology. Compared to more conventional radars operating in the microwave range, many components and subsystems which are required for sub/millimetre wave radars have to be specially developed as they are not yet readily available as commercial-off-the-shelf items. This paper highlights some enabling technologies for high performance radars operating in the sub/millimetre wave range. The author has been developing sub/millimetre wave radar systems for the past 15 years for applications in remote sensing and security. Of the dozen prototypes and advanced concept demonstrator radars he has developed, which span 24 to $340 \mathrm{GHz}$, this paper also presents some example radar systems which demonstrate the advantages afforded by these key enabling technologies.

\section{Enabling technologies}

This section presents some key technologies which have developed to enable the development of a range of high performance sub/millimetre wave radars.

\subsection{Chirp Generation}

Most commonly, sub/millimetre wave radars use frequency modulated continuous wave (FMCW) modulation which is compatible with the available solid-state signal generation technology. Typically, radars employ solid-state frequency multiplication, using MMIC or Schottky multipliers, driven by chirp generators with outputs in the microwave range. For

The 19th International Radar Symposium IRS 2018, June 20-22, 2018, Bonn, Germany 
high performance, chirps must be generated with high linearity and low phase noise and for many applications, high speed as well.

Chirp generation can be achieved in many ways but two of the most relevant are (i) a microwave voltage controlled oscillator (VCO) controlled by a phase locked loop (PLL) and (ii) a direct digital synthesiser (DDS) to generate the chirp directly which is upconverted onto a microwave stable local oscillator (STALO). The VCO/PLL option requires a simpler circuit and can be lower cost but may be limited in speed by the loop bandwidth, especially at the flyback points in a sawtooth modulation. The upconverted DDS option is inherently highly linear and offers the fastest dynamic response at the expense of a more complex (and more expensive) circuit. However, the most defining difference between these architectures is that the upconverted DDS exhibits inherently lower phase noise due to the use of fixed frequency oscillators for the STALO and DDS clock. A VCO at a similar frequency to the STALO will always have worse phase noise due to its inherent tenability. We have demonstrated that the upconverted DDS scheme can be between 10 and $30 \mathrm{~dB}$ lower phase noise than a VCO/PLL circuit, measured at $\sim 8 \mathrm{GHz}$ [7]. Superior phase noise is important for many applications including synthetic aperture radar, Doppler radar and vibrometry [8], [9].

\subsection{Feedhorns}

Antennas which couple between the waveguide of transmit and receive electronics and free space are essential components, either as feeds for primary antenna elements (lenses or reflectors) or as primary antennas in their own right. A variety of feedhorn designs may be employed in sub/millimetre wave radar depending on the system requirements and level of performance needed, with key parameters including beamwidth, sidelobe level, crosspolarisation and Gaussicity (power coupling to the fundamental free-space Gaussian mode).

For applications which require very low sidelobe levels, corrugated horns which carefully control the first three $\mathrm{HE}_{\mathrm{nn}}$ modes can achieve sidelobes at the $-60 \mathrm{~dB}$ level and a Gaussicity as high as $99.9 \%$, demonstrated at $94 \mathrm{GHz}$ [10]. At higher frequencies and for array applications where a large number of horns is needed, directly machined feedhorns in E-plane split-block offer a cost-effective alternative to corrugated horns. An array of 16 feedhorns with a smooth-walled spline profile was manufactured with this technique for use in a 340 $\mathrm{GHz}$ 3D imaging radar. All units showed excellent agreement with simulations over the design bandwidth of $30 \mathrm{GHz}$ and achieved a Gaussicity of over 99.2\% [11].

Reducing the complexity and cost of manufacture is a key driver for future radars and novel techniques such as additive manufacture offer significant promise. We have demonstrated excellent results from feedhorns with simple triple-linear profiles, after [12], which can be directly turned on a lathe. Fig. 1 shows such a feedhorn for use at $94 \mathrm{GHz}$ manufactured in brass with integral rectangular to circular transition. The aperture radius is $6.5 \mathrm{~mm}$, the length is $32 \mathrm{~mm}$ and the directivity is $19.2 \mathrm{dBi}$. The measured performance agrees well with the predictions obtained from the CORRUG mode matching software. This simple, low cost design achieve excellent mainlobe symmetry with low sidelobes $(-30 \mathrm{~dB})$ and low cross polar $(-40 \mathrm{~dB})$ in a compact form factor. Such a simple internal profile could be suitable for fabrication using additive manufacturing. 

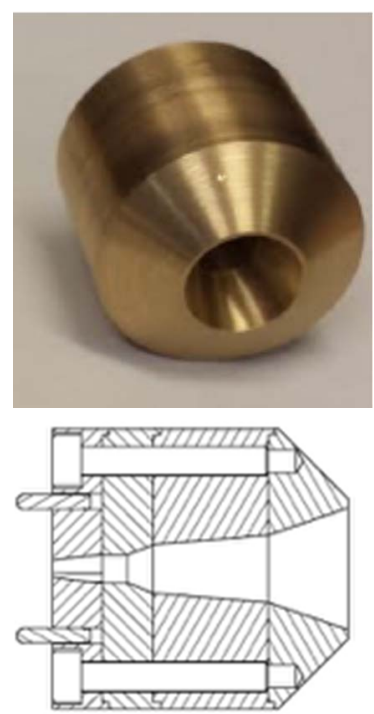

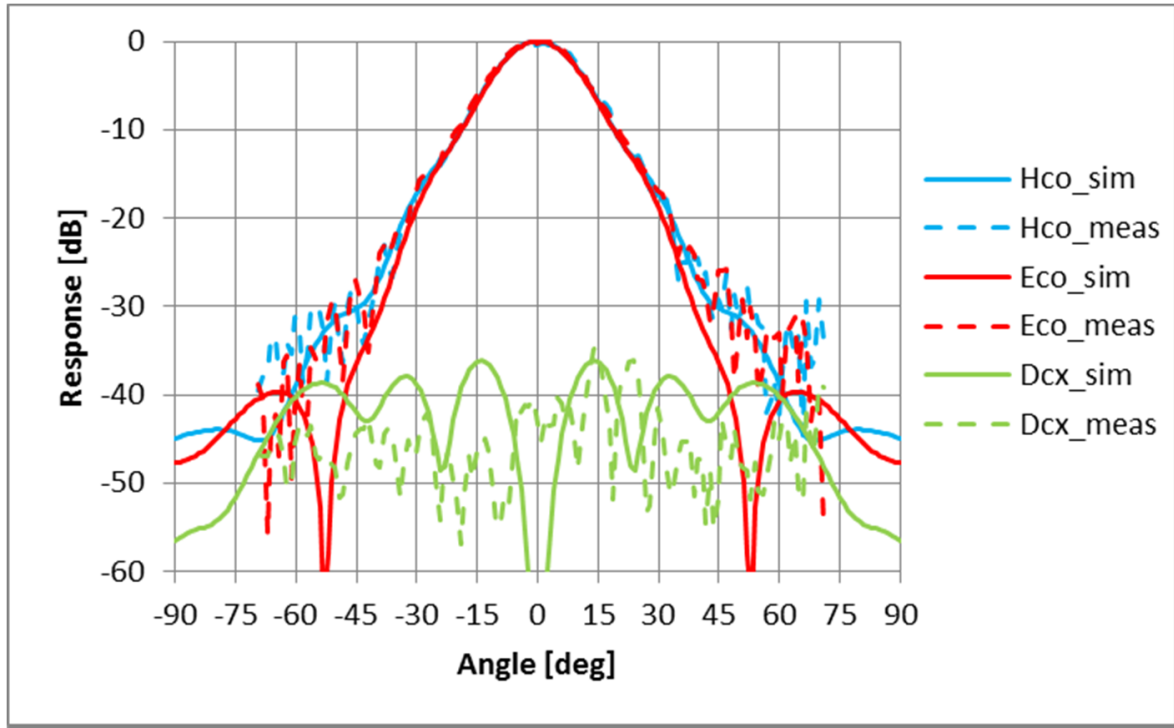

Figure 1. Triple-linear profile directly-turned $94 \mathrm{GHz}$ feedhorn (top left), cross section (bottom left) and measured results compared with CORRUG simulations (right).

\subsection{Duplexing}

Radars which must be configured to use a single antenna, typically due to space constraints, are forced to utilise a duplexer to separate transmit from receive signals. In waveguide this is typically done with a ferrite junction circulator for linear polarisation or sometimes an orthomode transducer with polariser for circular polarisation. In single antenna CW radars, the critical parameter in a duplexer is the transmit-receive leakage since any leaked signals tend to desensitise the receiver. Whilst methods exist to perform active or passive cancellation of leakage power, they are difficult to implement at sub/millimetre wave frequencies. Typical duplexing devices struggle to achieve isolation and leakage of better than about $-40 \mathrm{~dB}$ which is insufficient for high performance applications which seek to achieve receiver noise limited sensitivity.
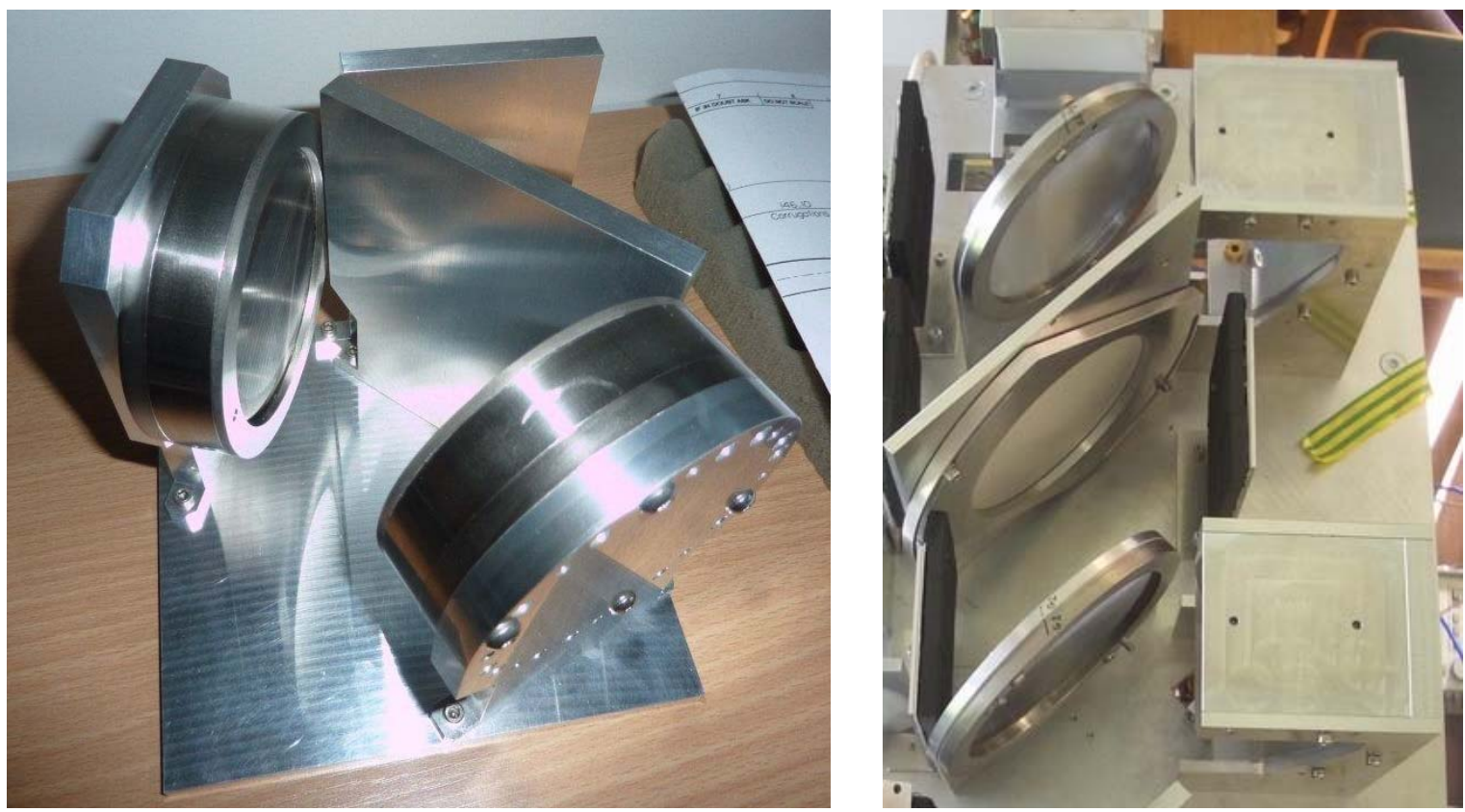

Figure 2. Frequency-selective polariser (FSP) based quasi-optical duplexer for $340 \mathrm{GHz}$ (left) and quasi-optical Faraday rotator based duplexer for $340 \mathrm{GHz}$ (right). 
An alternative approach, which offers higher levels of performance at the expense of greater size/weight, is to use a quasi-optical duplexer, which can be realised in different forms. A simple dielectric beamsplitter offers high isolation and low leakage but inherently adds a $3 \mathrm{~dB}$ insertion loss [13]. For linear cross-polarised operation a wire grid polariser offers excellent performance, for example better than $-80 \mathrm{~dB}$ at $94 \mathrm{GHz}$ (at the limit of VNA measurement) [14]. For circularly polarised operation variations on quarter-wave plates (both transmission and reflection forms) may be used including gratings [15]. Polarising interferometers may also be used such as the Martin-Puplett interferometer and the frequency-selective polariser (FSP) based duplexer [16]. For frequencies up to about $350 \mathrm{GHz}$ non-reciprocal free-space Faraday rotators made with permanently magnetised hexaferrite materials offer good isolation with low loss and if configured well can achieve very low leakage, for example $-80 \mathrm{~dB}$ at 340 GHz [17]. Fig. 2 shows an FSP based duplexer and a quasi-optical Faraday rotator based duplexer, both for $340 \mathrm{GHz}$ operation.

\subsection{Non-mechanical Beam Steering}

As more real-beam radar imaging applications push the requirement towards video frame rates, traditional mechanical beam steering solutions become inadequate and alternative solutions are required. The photo-injected Fresnel zone plate antenna (piFZPA) is one optoelectronic approach to non-mechanical beam steering [18]. The piFZPA is a reconfigurable Fresnel zone plate formed from an optically excited plasma within a semiconductor substrate, such as silicon. Optical reconfiguration of the zone plate masking pattern, which induces the required plasma characteristics in the semiconductor substrate, then permits rapid non-mechanical beam forming, focussing and steering at sub/millimetre wave frequencies.

We have demonstrated beam steering with piFZPAs at 94 and $188 \mathrm{GHz}$ with apertures up to $150 \mathrm{~mm}$ diameter [19], [20], [21]. Beam steering at rates of up to 17,000 beams per second was achieved using high speed digital micro-mirror devices (DMDs). This steerable antenna was utilised in a radar imaging demonstration at $94 \mathrm{GHz}$ and achieved 2D (PPI) imagery at 40 $\mathrm{Hz}$ frame rate and 3D volumetric imagery at $7 \mathrm{~Hz}$ frame rate. Fig. 3 shows example zone plate masks and simulations of off-axis far-field patterns.
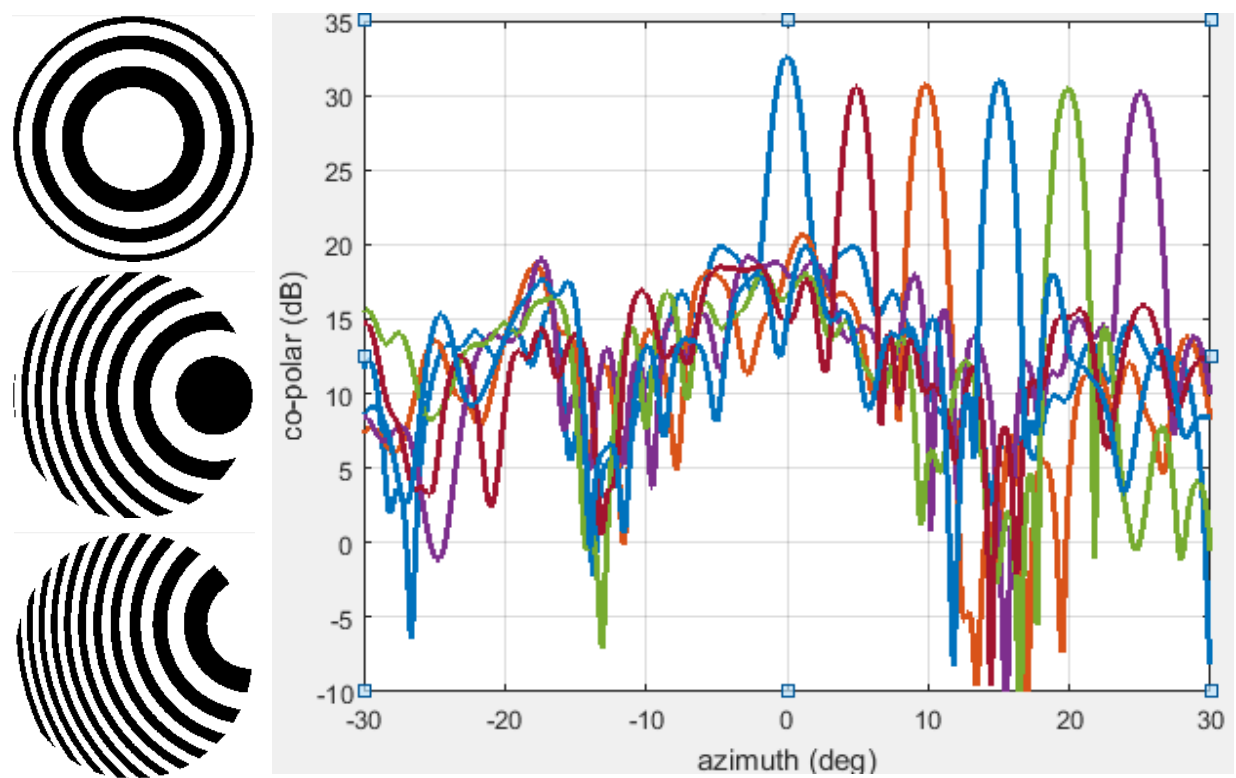

Figure 3. Photo-injected Fresnel zone plate antenna (piFZPA) zone mask examples (left) and simulated far-field patterns for off-axis beam steering (right). 


\section{Radar System Examples}

In order to illustrate some of the advantages afforded by using the types of enabling technologies shown above, this section presents some example sub/millimetre wave radar systems developed by the author and colleagues.

\section{1 "T-220" $94 \mathrm{GHz}$ coherent instrumentation radar}

The T-220 radar is a $94 \mathrm{GHz}$ FMCW instrumentation radar which is used for various data collection tasks and phenomenology studies [9]. It is fully coherent and hence useful for Doppler and vibrometry work. The radar uses separate transmit and receive antennas to achieve a high transmit-receive isolation of $70 \mathrm{~dB}$. Fig. 4 shows the radar and its system block diagram.

This radar makes use of the very low phase noise upconverted DDS chirp generation approach described in section 2.1 above. Chirps are generated by DDS at $200 \mathrm{MHz}$ and upconverted onto a $7.633 \mathrm{GHz}$ stable local oscillator (STALO) before being multiplied by 12 in a MMIC multiplier chain to $94 \mathrm{GHz}$. The maximum chirp bandwidth is $1.8 \mathrm{GHz}$ yielding range bins as fine as $8.3 \mathrm{~cm}$. The transmitted power is $+18 \mathrm{dBm}$ and the receiver noise figure is $6 \mathrm{~dB}$, providing high sensitivity and a wide dynamic range. The radar is well calibrated in amplitude for RCS measurements.

The radar is fully coherent and exhibits very low phase noise. Reflected transmitter phase noise from high RCS targets is not detectable in an $80 \mathrm{~dB}$ dynamic range, revealing low RCS targets in the same line of sight as high RCS targets. This is beneficial for surveillance and perimeter security applications. Additionally, the high sensitivity is advantageous when characterizing the signatures of low RCS targets and clutter.
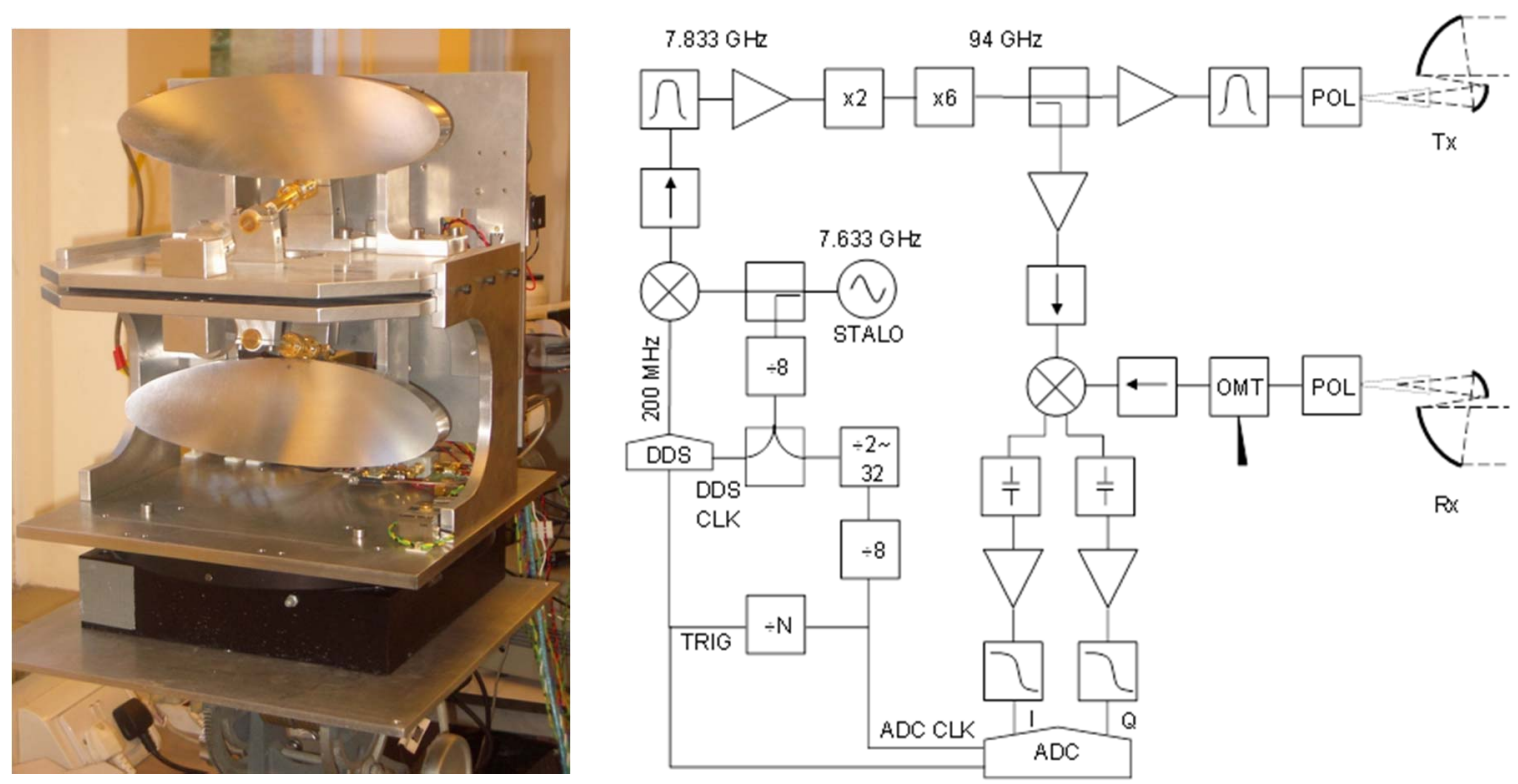

Figure 4. T-220 94 GHz radar (left) and system block diagram (right).

All timing signals (DDS clock, sampling clock, chirp repetition rate) are derived purely by division directly from the single master STALO. This results in excellent coherence and a high Doppler sensitivity so the radar is particularly useful for characterising the Doppler and micro-Doppler signatures of targets. Doppler measurements can be made in either $\mathrm{CW}$ or 
FMCW-Doppler (range-Doppler) mode. Fig. 5 shows an example velocity:time spectrogram of a manoeuvring DJI Phantom 3 Standard drone measured at $94 \mathrm{GHz}$ in FMCW-Doppler mode. The bulk Doppler of the drone and its changes in radial direction are clearly evident, as is the period during which the bulk Doppler was aliased between 3 and 5.5 seconds. The micro-Doppler signature due to the rapidly rotating propellers is evident as an extended Doppler structure on either side of the bulk signal. Such micro-Doppler signatures can be used to classify targets [22] in surveillance and security applications.

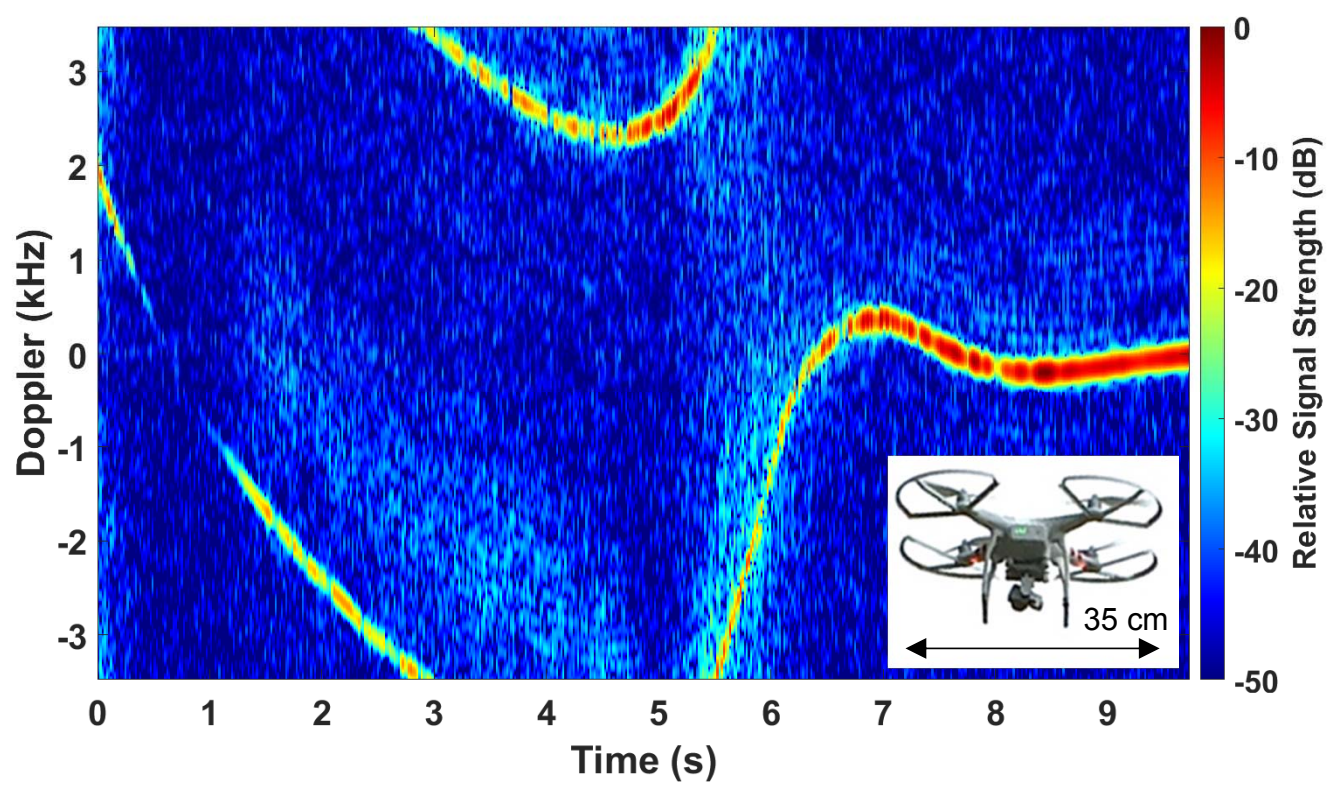

Figure 5. Velocity:time spectrogram of manoeuvring DJI Phantom 3 Standard drone (inset) measured at $94 \mathrm{GHz}$ in FMCW-Doppler mode.

\section{$\underline{3.2 \text { "CONSORTIS" } 340 \mathrm{GHz} \text { 3D imaging radar }}$}

Submillimetre wave imaging is a promising modality for future security screening applications due to its ability to see through clothing with high resolution. The EU FP7 CONSORTIS project (Concealed Object Stand-Off Real-Time Imaging for Security) has developed a demonstrator system for next generation airport security screening which combines passive and active submillimetre wave imaging sensors. The author led the team which developed the CONSORTIS radar subsystem: a 16-channel, $340 \mathrm{GHz}$ FMCW radar which produces $3 \mathrm{D}$ maps of the subject with $\sim 1 \mathrm{~cm}^{3}$ voxel resolution over a $1 \mathrm{~m}^{3}$ sense volume at multi-hertz frame rates [23], [24]. The radar combines a spare focal plane array (FPA) of 16 transceiver channels with state-of-the-art wide field of view quasi-optics and mechanical beam steering to achieve the wide field-of-view, high volumetric resolution imaging at frame rates of up to $10 \mathrm{~Hz}$. The radar subsystem is shown in Fig. 6 along with a ray diagram of the optical elements, transceiver FPA and corresponding target image plane.

To achieve the high performance required, the CONSORTIS radar embodies a number of significant enabling technologies. These include a wideband upconverted DDS chirp generator based on the architecture described in section 2.1 and 16 E-plane split-block manufactured smooth-walled, spline profile feedhorns, as mentioned in section 2.2, which achieve both good near field and far-field performance. 

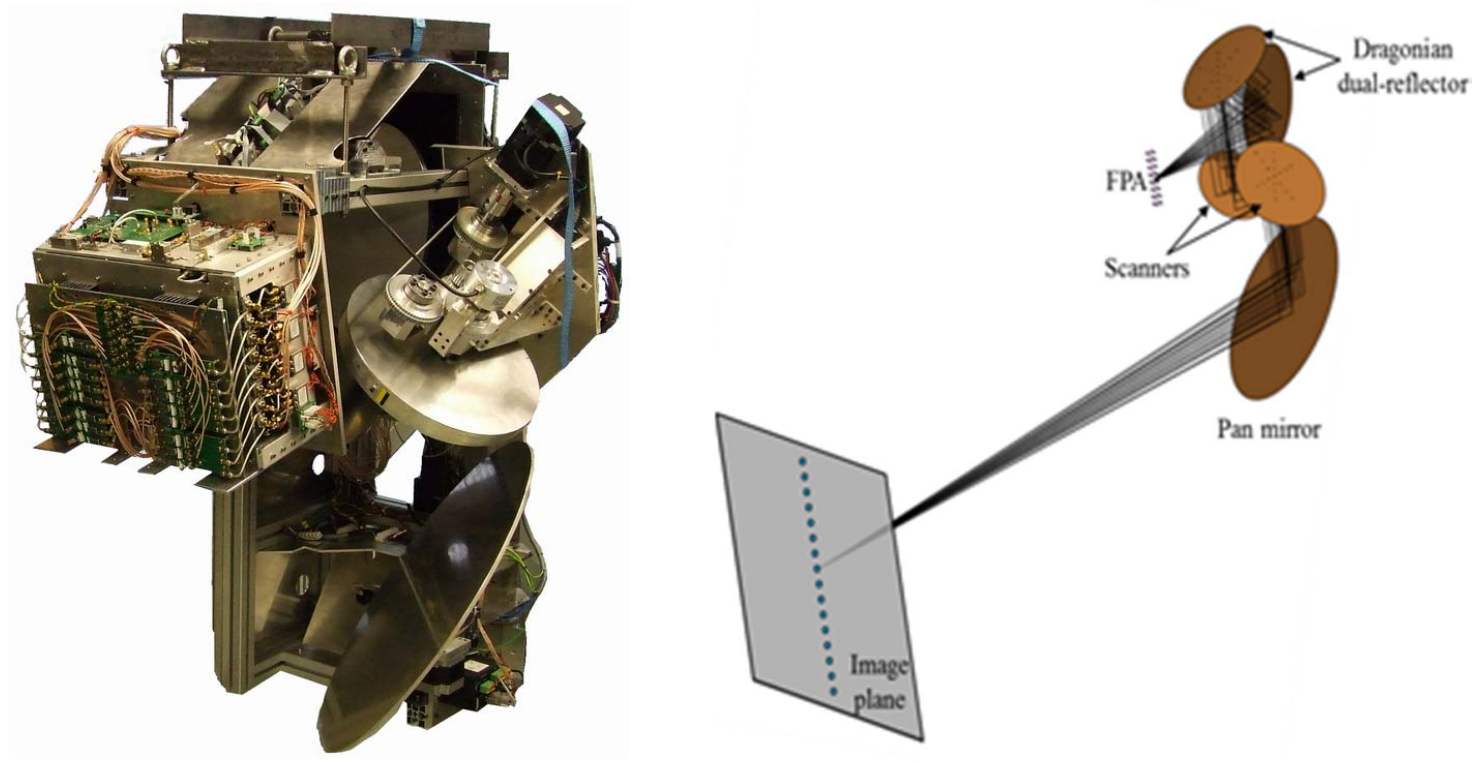

Figure 6. CONSORTIS radar subsystem (left)

and ray diagram of optical elements with transceiver array and $1 \times 1 \mathrm{~m}^{2}$ target focal plane (right).

Two advanced technologies developed by project partners [25] were employed in the signal chain which brought multiple advantages to the radar subsystem. Firstly, single chip GaAs MMIC x8 frequency multipliers were developed which provided efficient, wideband multiplication of the X-band chirp generator output to $\sim 85 \mathrm{GHz}$ for subsequent multiplication by two cascaded Schottky doublers to $340 \mathrm{GHz}$. The whole multiplier chain exhibits very good group delay behaviour such that the chirp linearity is maintained and the radar raw point response is barely degraded from the ideal case. Secondly, the final Schottky doubler stage was configured to also act as a subharmonic mixer in receive such that reflected FMCW signals are deramped and the IF signal is extracted via the bias line. This self-mixing multiplier architecture completely avoids the need for explicit duplexing components and made it feasible to close pack the transceiver blocks into a 16 channel array.

The balance in total FMCW radar signal budget across all 16 channels (accounting for transmit power, conversion loss and IF gain variations across the $30 \mathrm{GHz}$ chirp bandwidth) is a little over $3 \mathrm{~dB}$ which is considered excellent at this carrier frequency. To maintain real-time operation, the signal processing must not lag the input data rate, which is $147.4 \mathrm{M}$ samples per second at $10 \mathrm{~Hz}$ frame rate. The processor thus has to capture and compute $14,4001 \mathrm{k}-$ point FFTs within $100 \mathrm{~ms}$. This is achieved using a parallelised multi-threaded $\mathrm{C}$ code running on a mid-level desktop PC. FMCW processing and intensity image rendering are handled on the radar control PC in real time. Thresholded and truncated data are transferred to a separate PC for subsequent analysis of the imagery to identify anomalies which may indicate the presence of concealed objects.

The radar is mounted in the CONSORTIS system enclosure, which also includes the passive submillimetre wave camera developed by the project consortium, and controlled automatically by a passenger control subsystem which maintains passenger flow and ensures passengers are scanned one at a time.

Fig. 7 shows an example visible image of the author as viewed by the radar alongside the corresponding raw image frame of maximum intensity obtained with the radar. Note the boresights of the two sensors were not co-aligned (the camera was mounted below the radar) 
so the perspective views do not perfectly match. The radar image presents the maximum intensity per line of sight in the image encoded over a $60 \mathrm{~dB}$ colour scale from dark purple to white. The banding in the background is due to differences in the noise floor of some channels. The high spatial resolution is evident, clearly revealing features such as folds in the clothing and the trouser belt. The bright highlight on the chest is due to a mobile phone carried in the shirt pocket. The reflected power from such a planar metallic object is so high that it clips the colour scale to green as a warning. Note also the presence of bright reflections from clothing which are typical at these frequencies. In practice, the full $3 \mathrm{D}$ volumetric information available from the radar range profiles must be used for concealed anomaly detection to 'see through' the outer clothing.

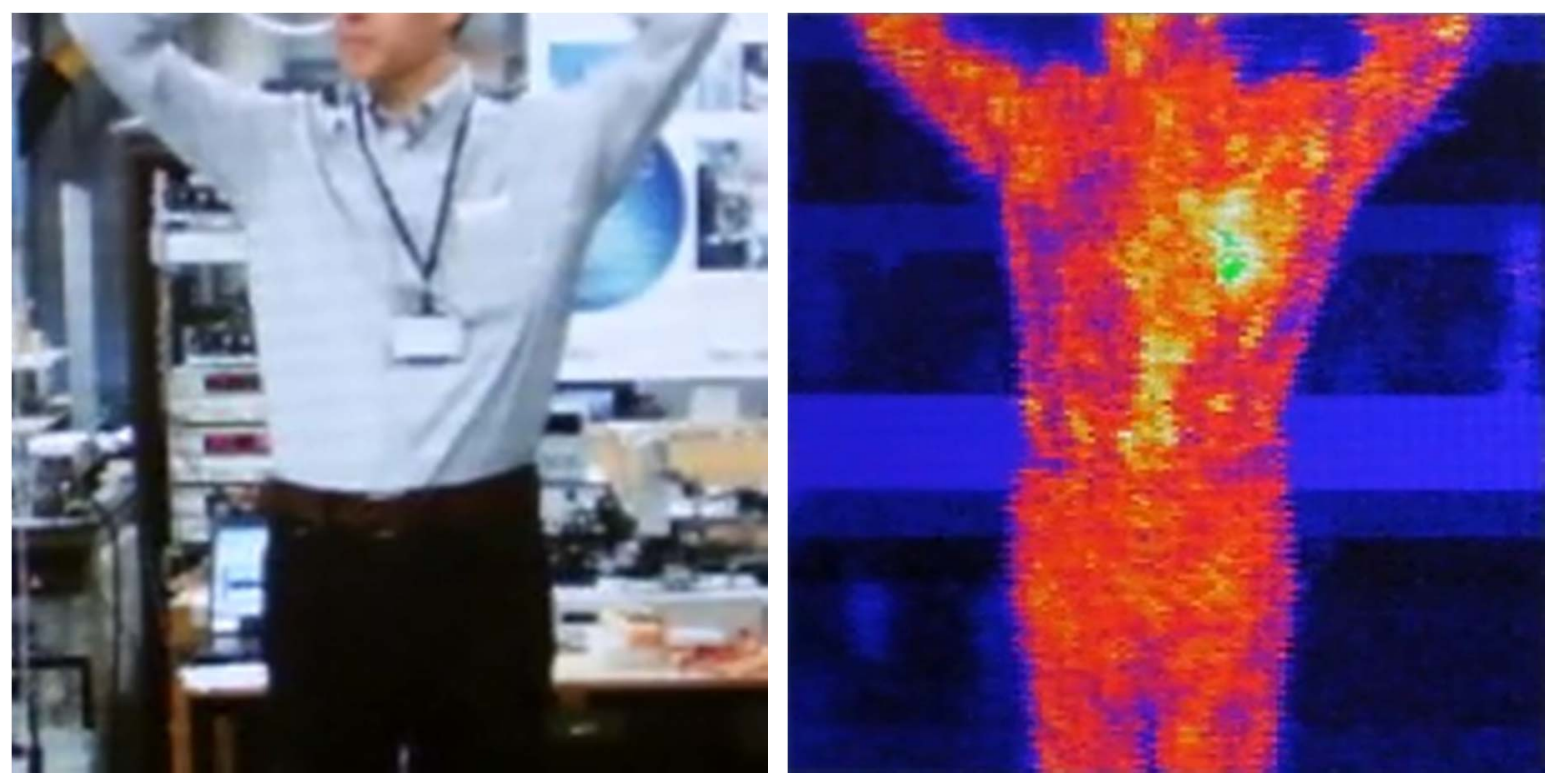

Figure 7. Example visible image of the author as viewed by the radar (left) and corresponding raw image frame of maximum intensity obtained by the radar ( $60 \mathrm{~dB}$ colour scale) (right).

\section{Conclusions}

The relative commercial immaturity of certain components for sub/millimetre wave radar has driven the development of key enabling technologies. This paper has presented some examples covering chirp generation, feedhorns, duplexing and non-mechanical beamsteering. The resulting advantages afforded by such technologies have been illustrated by the description of two example high performance radars systems: firstly, the T-220 $94 \mathrm{GHz}$ instrumentation radar which exhibits very low phase noise and excellent coherence, and secondly the CONSORTIS $340 \mathrm{GHz} 16$ channel 3D imaging radar which is capable of mapping human subjects at multi-hertz frame rates with $\sim 1 \mathrm{~cm}^{3}$ voxel resolution.

Future sub/millimetre wave radar systems will see continued improvement through developments in enabling technologies. The performance of semiconductor devices continues to improve at ever higher frequencies and there is a strong trend towards greater levels of electronic integration. This will yield more compact systems and reduce costs, both of which are vital if sub/millimetre wave radars are to become commercially viable. Novel fabrication methods such as additive manufacturing are developing rapidly and if dimensional tolerances and surface finish continue to improve, we will see an increase in the utilisation of these methods in the production of components for radars at high frequencies. One technology which is critical to enabling many future radar systems is non-mechanical beam steering. 
Mechanical beam steering whilst feasible has its limitations (speed, cost, size, reliability, noise etc.) and there is a need for further research into practical non-mechanical beam steering alternatives of all forms (electronic, optoelectronic, computational, etc.).

The field of sub/millimetre wave radar development appears vibrant and growing and the diverse range of applications for such radars is also increasing. With the continued development of novel technologies, the advantages offered by sub/millimetre wave radars will continue to expand, providing high quality sensing for a wider range of end users. The enabling technologies outlined in this paper also have potential application in other fields such as vacuum tube gyro-amplifiers and oscillators (feedhorns), communications (feedhorns, non-mechanical beam steering), spectroscopy (chirp generator, feedhorns, duplexing) and plasma diagnostics (chirp generator, feedhorns, duplexing, non-mechanical beam steering).

\section{Acknowledgements}

The author sincerely thanks his many colleagues and collaborators who have contributed to the work reported in this paper and to the many agencies which have provided funding.

\section{References}

[1] Cooper, K.B. and Chattopadhyay, G., "Submillimeter-Wave Radar: Solid-State System Design and Applications," IEEE Microwave Magazine, 15, (7), pp. 51-67, 2014.

[2] Quast, H. and Loffler, T., "3D-terahertz-tomography for material inspection and security," in Proc. 34th Int. Conf. Infrared, Millimeter, Terahertz Waves, pp. 1-2, 2009.

[3] Goshi, D.S. and Bui, L.Q., "Power line characterization from an airborne data collection with a millimeter wave radar," Proc. SPIE 9077, Radar Sensor Technology XVIII, 2014.

[4] Ziegler, V., Schubert, F.,Schulte, B., Giere, A., Koerber, R. and Waanders, T., "Helicopter Near-Field Obstacle Warning System Based on Low-Cost Millimeter-Wave Radar Technology", in IEEE Trans. Microwave Theory Tech., 61, (1), pp. 658-665, 2013.

[5] Im, E., Wu, C., and Durden, S.L., "Cloud profiling radar for the CloudSat mission," Proc. IEEE Int. Radar Conf., pp. 483-486, 2005.

[6] Cooper, K.B. et al., "Atmospheric Humidity Sounding Using Differential Absorption Radar Near 183 GHz”, IEEE Geosci. Rem. Sens. Letters, vol. 15, no. 2, pp. 163-167, Feb. 2018.

[7] Robertson, D.A., Cassidy, S.L. \& Bolton, D.R., "Nonlinearity and phase noise effects in 340 $\mathrm{GHz}$ 3D imaging radar", Proc. SPIE 8715, Passive and Active Millimeter-Wave Imaging XVI, 87150M, 2013

[8] Robertson, D.A. \& Cassidy, S.L., "Micro-doppler and vibrometry at millimeter and submillimeter wavelengths", Proc. SPIE 8714, Radar Sensor Technology XVII, 87141C, 2013

[9] Robertson, D.A., Brooker, G.M. \& Beasley, P.D.L., "Very low-phase noise, coherent $94 \mathrm{GHz}$ radar for micro-Doppler and vibrometry studies", Proc. SPIE 9077, Radar Sensor Technology XVIII, 907719, 2014

[10] McKay, J., Robertson, D.A., Speirs, P.J., Hunter, R.I., Wylde, R. \& Smith, G.M., "Compact corrugated feedhorns with high Gaussian coupling efficiency and ultra-low sidelobes", IEEE Trans. Antennas \& Propagation, 64, (6), pp. 2519-2522, 2016

[11] Robertson, D.A., Macfarlane, D.G., Hunter, R.I., Cassidy, S.L., Llombart, N., Gandini, E., Bryllert, T., Ferndahl, M., Lindström, H., Tenhunen, J., Vasama, H., Huopana, J., Selkälä, T., Vuotikka, A-J., "High resolution, wide field of view, real time $340 \mathrm{GHz}$ 3D imaging radar for 
security screening", Proc. SPIE 10189, Passive and Active Millimeter-Wave Imaging XX; 101890C, 2017

[12] Granet, C., Bolton, R., and Moorey, G., "A smooth-walled spline-profile horn as an alternative to the corrugated horn for wide band millimeter-wave applications," IEEE Trans. Antennas Propag., vol. 52, no. 3, pp. 848-854, 2004

[13] Cooper, K.B. et al, "THz Imaging Radar for Standoff Personnel Screening”, IEEE Trans Terahertz Sci. Tech., 1 (1), pp. $169-182,2011$

[14] Monje, R.R., Cooper, K.B., Dengler, R.J., El Bouyadi, T.O. \& Gonzalez-Ovejero, D., "High T/R Isolation and Phase Noise Suppression in Millimeter wave FMCW Radars", IRMMW, 2017

[15] Cooper, K.B., Llombart, N., Chattopahyay, G., Dengler, B., Cofield, R.E., Lee, C., Fichenkov, S. \& Koposova, E., "A Grating-Based Circular Polarization Duplexr for Submillimeter-Wave Transceivers", IEEE Microwave Wireless Comp. Lett., 22 (3), pp. 108 - 110, 2012

[16] Manabe, T., Inatani, J., Murk, A., Wylde, R.J., Seta, M. \& Martin, D.H., "A new configuration of polarization-rotating dual-beam interferometer for space use", IEEE Trans. Microwave Theory Tech., 51 (6), pp. 1696 - 1704, 2003

[17] Robertson, D.A., Marsh, P.N., Bolton, D.R., Middleton, R.J.C., Hunter, R.I., Speirs, P.J., Macfarlane, D.G., Cassidy, S.L., and Smith, G.M., "340 GHz 3D radar imaging test bed with $10 \mathrm{~Hz}$ frame rate.", Passive and Active Millimeter-Wave Imaging XV, Proc. SPIE 8362, 836206, 2012

[18] Webb, G.W., and Pinck, L.H., "Light-controlled MMW beam scanner," SBMO International Microwave Conference/Brazil, 2, pp. 417 - 422, 1993

[19] Gallacher, T.F., Robertson, D.A. and Smith, G.M., "The Photo-Injected Fresnel Zone Plate Antenna: Optoelectronic Beam Steering at mm-Wave Frequencies," IEEE Trans. Antennas Propag., 61(4), pp. 1688 - 1696, 2013

[20] Gallacher, T.F., Sondena, R., Robertson, D.A. and Smith, G.M., "Off-Axis Measurements on a mm-Wave Optically Controlled Lens Antenna," IEEE Antennas and Wireless Propagation Letters, 12 , pp. $1520-1522,2013$

[21] Robertson, D. A., Gallacher, T., Sondena, R. \& Macfarlane, D. G., "Millimeter wave imaging at up to 40 frames per second using an optoelectronic photo-injected Fresnel zone plate lens antenna", Proc. SpIE 9830, Passive and Active Millimeter-Wave Imaging XIX. pp. 1-9, 2016

[22] Rahman, S. \& Robertson, D.A., "Time-Frequency Analysis of Millimeter-Wave Radar MicroDoppler Data from Small UAVs", Sensor Signal Processing for Defence Conference, pp. 1 5,2017

[23] Robertson, D.A. et al, "High resolution, wide field of view, real time 340GHz 3D imaging radar for security screening", Proc. SPIE 10189, Passive and Active Millimeter-Wave Imaging XX, 101890C, 2017

[24] Robertson, D.A. et al, "A high frame rate, $340 \mathrm{GHz} 3 \mathrm{D}$ imaging radar for security", IEEE Radar Conf., 2018.

[25] Dahlbäck, R., Bryllert, T., Granström, G., Ferndahl, M. Drakinskiy, V. and Stake, J., "Compact $340 \mathrm{GHz}$ homodyne transceiver modules for FMWC imaging radar arrays," IEEE MTT-S International Microwave Symposium (IMS), pp. 1 - 4, 2016 